\title{
SRPX2 promotes cell proliferation and invasion via activating FAK/SRC/ERK pathway in non-small cell lung cancer
}

\author{
Xiujuan $\mathrm{Li}^{1 *}$, Jing Liu ${ }^{1 *}$, Hong Sun ${ }^{\square}$, Yong Zou ${ }^{2}$, Juan Chen ${ }^{3}$, Yichun Chen ${ }^{4}$, Cheng Chen ${ }^{1}$ \\ and Xuan Wu'
}

'Departmen of Geriatrics, Wuhan NO. 1 Hospital, Wuhan, Hubei, China; ${ }^{2}$ Department of Emergency, Wuhan Medical Emergency Center, Wuhan, Hubei-China; ${ }^{3}$ Wuhan Hankou Hospital-Wuhan, Hubei, China; ${ }^{4}$ Department of Digestive, Wuhan NO. 8 Hospital, Wuhan, Hubei, China

\begin{abstract}
Background: Recent studies showed that sushi repeat containing protein $X$ linked 2 (SRPX2) could participate in the development of various malignant tumors. However, its role in non-small cell lung cancer (NSCLC) was unknown. The aim of the study was to prospectively investigate the role of SRPX2 in NSCLC cell proliferation, migration and invasion and reveal the underlying mechanism. Material and methods: Quantitative real-time polymerase chain reaction ( $\mathrm{QRT}$-PCR), western blot and immunohistochemistry - IHC) were used to measure detect the mRNA and protein levels, respectively, in NSCLC tissues and cell lines. Cell Counting Kit-8 (CCK-8), colony formation, wound healing and transwell assays were utilized to assess cell proliferation, migration and invasion. In vivo subcutaneous xenograft tumor model was established to detect the tumorigenic function of SRPX2, and IHC assay was performed to measure protein expression. Results: SRPX2 expression was upregulated in NSCLC tissues and cell lines, and positively correlated with tumor size, lymph node metastasis, distant metastasis and clinical stage. High SRPX2 expression also predicted poor prognosis. In vitro experiments indicated that overexpression of SRPX2 promoted the proliferation, migration, and invasion of SPC-A1 cells while knockdown of SRPX2 caused the opposite effects in A549 cells. Specifically, SRPX2 activated FAK/SRC/ERK pathway and its downstream effectors and promoted epithelial-mesenchymal transition (EMT). Conclusion: Taken together, our findings revealed a functional role of SRPX2 in NSCLC cell proliferation, migration and invasion. The underlying mechanism was, at least partially, the activation of FAK/ SRC/ERK pathway. This study provides the molecular basis for targeting SRPX2 in potential clinical application for NSCLC.
\end{abstract}

Key words: SRPX2; NSCLC; proliferation; migration; invasion; FAK/ SRC/ERK

Received: 10 January, 2020; revised: 20 April, 2020; accepted: 20 May, 2020; available on-line: 18 June, 2020

\footnotetext{
घe-mail: HongSudgh@163.com

*These authors contributed equally

Acknowledgements of Financial Support: This study was supported by Medical Research Projects of Wuhan in 2019, No.WZ19C28, Medical Research Projects of Wuhan in 2017, No.WZ17D01.

Abbreviations: NSCLC, non-small cell lung cancer; SRPX2, sushi repeat-containing protein X-linked 2; FAK, focal adhesion kinase; ERK, extracellular signal-regulated kinase; MAPK, mitogen-activated protein kinase; qRT-PCR, Quantitative real-time polymerase chain reaction; IHC, Immunohistochemistry; CCK-8, Cell Counting Kit-8; EMT, epithelial-mesenchymal transition; ECM, extracellular matrix
}

\section{INTRODUCTION}

Lung cancer has one of the world's highest incidence and mortality among the malignant tumors, and is the leading cause of cancer death and poses a serious threat to human life and health (Jemal et al., 2010). Lung cancer can be divided into two categories according to its pathological features: small-cell lung cancer (SCLC) and non-small cell lung cancer (NSCLC, including adenocarcinoma, large-cell carcinoma, squamous cell carcinoma and adenosquamous carcinoma) (Youlden \& Baade, 2008). In fact, NSCLC is the major type of lung cancer accounting for about $80 \%$ to $85 \%$ of the total lung cancer cases (Gridelli et al., 2015). However, due to the lack of molecular markers for early diagnosis, more than half of NSCLC patients suffer from late clinical diagnosis, leading to cancer cell invasion and metastasis. The five-year overall survival (OS) rate is only about $17 \%$ (Gu et al., 2016). Therefore, studying the pathogenesis of NSCLC and finding efficient early diagnostic markers are critical for the discovery of therapeutical targets and improving OS rate.

The local microenvironment of cancer cells plays an indispensable role in the progression of cancer. The main component of the microenvironment is the extracellular matrix (ECM), which mainly includes glycoproteins, proteoglycans and collagen (Caterson, 2012). These molecules are important for tissue and organ morphogenesis and for the maintenance of cell and tissue structure and function (Özbek et al., 2010; Rosso et al., 2004). The binding of ECM to cell surface receptors (such as integrins) can initiate a series of signaling cascades that control cell survival, differentiation, proliferation, invasion, migration, apoptosis etc. (Jin \& Varner, 2004; Rozario \& DeSimone, 2010; Wickström \& Fässler, 2011). Thus, it seems that ECM dysfunction is one of the important features in tumor progressions, and abnormal ECM can lead to the infiltration and metastasis of tumor cells (Venning \& Erler, 2015; Naba et al., 2014; Malandrino et al., 2018; Pickup \& Weaver, 2014; Lomberk, 2010. Sushi repeat containing protein X linked 2 (SRPX2) was firstly discovered in leukemia cells. It is a chondroitin sulfate proteoglycan (Kurosawa et al., 1999). Increasing evidence suggested that mutations of SRPX2 cause seizures (Royer-Zemmour et al., 2008; Salmi et al., 2013), linguistic and cognitive dysfunction (Roll et al., 2006), and mental retardation (Sia \& Huganir, 2013). Recent studies showed that SRPX2 protein can also participate in the development of a variety of malignant tumors including gastric cancer (Tanaka et al., 2009; Tanaka et al., 2012), colorectal cancer (Øster et al., 2013; Liu et al., 2015), pancreatic 
cancer (Gao et al., 2015) and glioblastoma (Tang et al., 2016). The accumulating evidence that SRPX2 is important for tumorigenesis and development suggests that SRPX2 may be a valuable new target for tumor treatment. Nevertheless, its function and molecular mechanism of action in NSCLC have not yet been elucidated.

In this study, we analyzed the expression of SRPX2 in NSCLC tissues and explored the correlation between SRPX2 expression and clinicopathological results. In addition, the role of SRPX2 in NSCLC cell proliferation, migration and invasion was analyzed both in the in vitro and in vivo experiments. Moreover, the potential regulatory mechanism in NSCLC was also proposed.

\section{MATERIALS AND METHODS}

Patients and Tissues. This study was performed in accordance to the tenets of the Declaration of Helsinki and the ethical guidelines for medical and health research of the National Institutes of Health $(\mathrm{NIH})$ and approved by the Ethics Committees of Wuhan NO.1 Hospital (Wuhan, Hubei Province, China). A total of 46 pairs of NSCLC tissue samples and matched adjacent normal tissue samples were obtained from patients who had surgery at Wuhan NO. 1 Hospital. All enrolled patients gave their written informed consent.

Immunohistochemistry (IHC) assay. NSCLC and adjacent normal tissue samples of the enrolled patients and mouse tumor tissues were fixed, embedded, sliced into 4-mm sections and incubated with primary antibodies: anti-SRPX2, anti-Ki-67, and anti-E-cadherin (Abcam, Cambridge, MA, USA) at $4^{\circ} \mathrm{C}$ overnight. Then, the sections were incubated with HRP-conjugated goat anti-rabbit IgG secondary antibody for $1 \mathrm{~h}$ at room temperature. The peroxidase was detected using 3,3'-diaminobenzidine tetrahydrochloride and counterstained with hematoxylin.

Cell culture. The normal human bronchial epithelial cell line (16HBE) and human NSCLC cell lines (A549, H1975, SPC-A1, H1229 and H460) were obtained from the Cell Bank of the Chinese Academy of Science (Shanghai, China). All the cell lines were maintained in Dulbecco's modified Eagle's medium (DMEM, Thermo Fisher Scientific, Inc., Waltham, MA, USA) containing 10\% fetal bovine serum (FBS, Thermo Fisher Scientific, Inc.), $100 \mathrm{U} / \mathrm{ml}$ penicillin (Beyotime Institute of Biotechnology, Shanghai, China) and $100 \mu \mathrm{g} / \mathrm{ml}$ streptomycin (Beyotime Institute of Biotechnology) at $37^{\circ} \mathrm{C}$ in a humidified incubator with $5 \% \mathrm{CO}_{2}$.

Cell transfection. For overexpression or knockdown of SRPX2, the pcDNA3.1-SRPX2, pcDNA3.1-NC, nontargeting shRNA (sh-NC) and shRNA targeting SRPX2 (sh-SRPX2) were obtained from Shanghai GenePharma Co, Ltd (Shanghai, China). Lipofectamine 2000 (Thermo Fisher Scientific, Inc.) was used for the transfection according to the manufacturer's protocols.

RNA extraction and qRT-PCR. Cells $\left(1 \times 10^{5}\right.$ cells/ well) transfected with indicated plasmids were seeded onto a 6-well culture plate and incubated for 24 hours. Total RNA was extracted from cells and tissues using TRIzol reagent (Thermo Fisher Scientific, Inc.) according to the manufacturer's instruction and then reversetranscribed using the PrimeScript RT Reagent Kit (Takara, Bio Inc., Shiga, Japan). PCR assays were performed in an ABI-7500 system (Applied Biosystems, Foster City, CA, USA) using the SYBR Premix Ex Taq GC kit (Takara, Bio Inc.). All samples were normalized to GAPDH and the expression was calculated using $2^{-\Delta \Delta \mathrm{Ct}}$ method. The following primers were used: SRPX2, 5'-TAGTG-
GCACTTACACCTGCAC-3' (forward), 5'-ATTCGGCTGCGATCACCTTC-3' (reverse); GAPDH, 5'-CTGCACCACCAACTGCTTAG-3' (forward), 5'-AGGTCCACCACTGACACGT'T-3' (reverse).

Western blot. Cells $\left(1 \times 10^{5}\right.$ cells/well $)$ transfected with indicated plasmids were seeded onto a 6-well culture plate and incubated for 24 hours. Proteins were extracted from tissues and cells using RIPA lysis buffer (Beyotime Institute of Biotechnology), and were separated by sodium dodecyl sulfate polyacrylamide gel electrophoresis (SDS-PAGE), and transferred to polyvinylidene fluoride (PVDF) membranes. After blocking with $5 \%$ nonfat milk for $1 \mathrm{~h}$, the membranes were incubated with primary antibodies as follows: anti-SRPX2 (1:1000, ab91584, Abcam), anti-PCNA (1:1000, ab92552, Abcam), anti-E-cadherin (1:500, ab15148, Abcam), anti$\mathrm{N}$-cadherin (1:1000, ab76057, Abcam), anti-FAK (1:2000, ab40794, Abcam), anti-phospho-FAK (Tyr576/577, 1:1000, \#3281, Cell Signaling Technology, Beverly, MA, USA), anti-Erk1/2 (1:1000, \#9102, Cell Signaling Technology), anti-phospho-Erk1/2 (Tyr202/Tyr204, 1:1000, \#4377, Cell Signaling Technology), anti-SRC (1:1000, \#2108, Cell Signaling Technology), anti-phospho-SRC (Tyr416, 1:1000, \#2101, Cell Signaling Technology) and anti-GAPDH (1:10000, ab181602, Abcam). Subsequently, membranes were probed with corresponding secondary antibody (Abcam), visualized by ECL reagent (Invitrogen) and detected using an imaging system (Bio-Rad, Hercules, CA, USA).

Cell counting kit-8 (CCK-8) assay. Briefly, 1000 cells/well were seeded onto 96-well plates and transfected. $10 \mu \mathrm{L}$ of CCK-8 assay (Dojindo Laboratories, Kumamoto, Japan) was used according to the manufacturer's instructions: added to each well and incubated for 2 hours. The absorbance was measured at $450 \mathrm{~nm}$ using a Microplate Autoreader (Thermo Fisher Scientific, Inc.) 24, 48, 72 and 96 hours after transfection.

Colony formation assay. 1000 cells/well were maintained in 6-well plates with DMEM medium replaced every 3 days for two weeks. The colonies were subsequently fixed in paraformaldehyde (PFA, Sigma-Aldrich, St. Louis, MO, USA) and stained in $0.5 \%$ crystal violet (Beijing Solarbio Science \& Technology Co.Ltd, Beijing, China). Stained cells were washed in PBS, then photographed using a microscope and counted (Olympus Corporation, Tokyo, Japan).

Wound healing assay. Cells $\left(1 \times 10^{5}\right.$ cells/well $)$ were cultured in 6-well plates to reach $85 \%$ confluence, and then mitomycin C $(10 \mu \mathrm{g} / \mathrm{ml}$, Sigma-Aldrich) was added to the cells for 2 hours. A wound was scratched in the cell monolayer in each plate with a $200 \mu \mathrm{l}$ pipette tip. The cell debris was removed by PBS wash and the remaining cells were maintained in serum-free medium. At $0 \mathrm{~h}$ and $24 \mathrm{~h}$ after the scratch, the images were recorded using a microscope (Olympus Corporation).

Transwell assay. $1 \times 10^{5}$ cells/well were seeded onto the upper chamber (Corning Life Sciences, Tewksbury, MA, USA) pre-coated with $100 \mu \mathrm{g}$ Matrigel (BD Biosciences, Bedford, MA, USA). $500 \mu \mathrm{l}$ of serum-free medium and $400 \mu \mathrm{l}$ of DMEM medium with $10 \%$ FBS were separately added into the upper and lower chamber, respectively. After 24 hours, cells in the lower chamber were fixed in $4 \%$ PFA, stained with $0.5 \%$ crystal violet and counted under a microscope (Olympus Corporation).

Tumor xenograft. The animal experiments were performed according to the guidelines for the care and use of laboratory animals with the approval of Wuhan NO.1 Hospital and the Guide for NIH and the institutional 
A

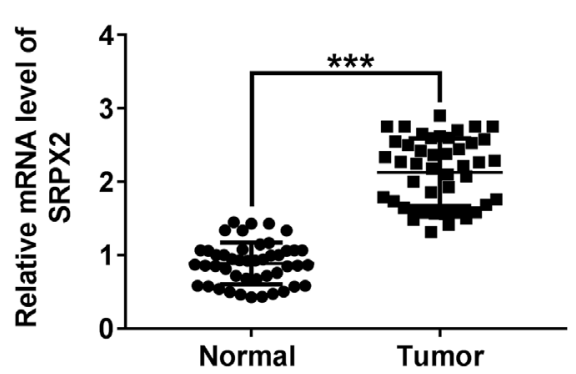

C
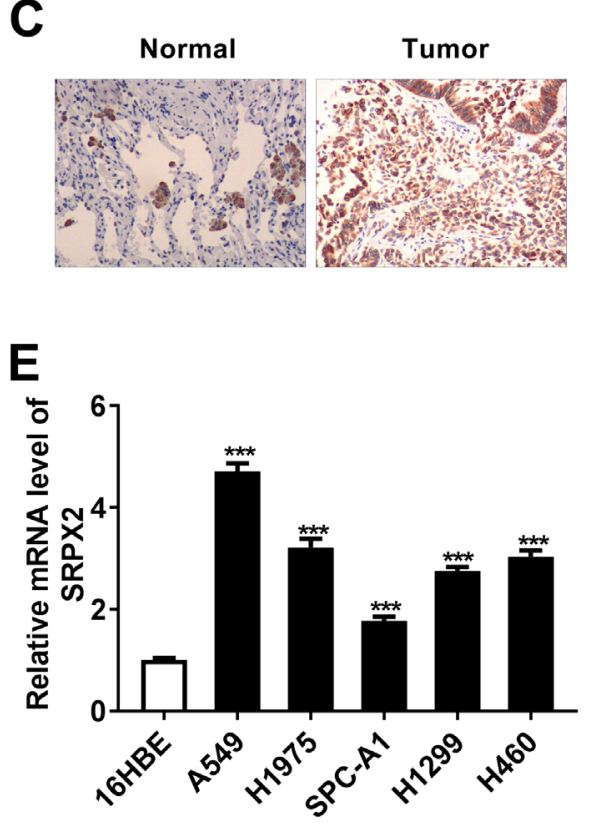

B

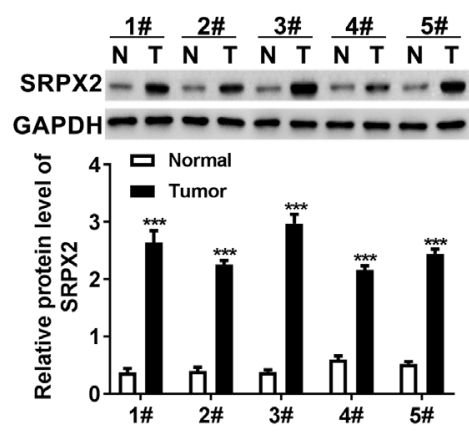

D

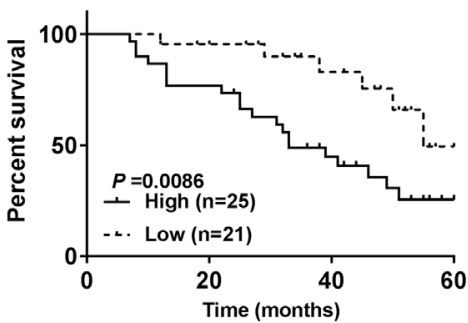

$\mathbf{F}$

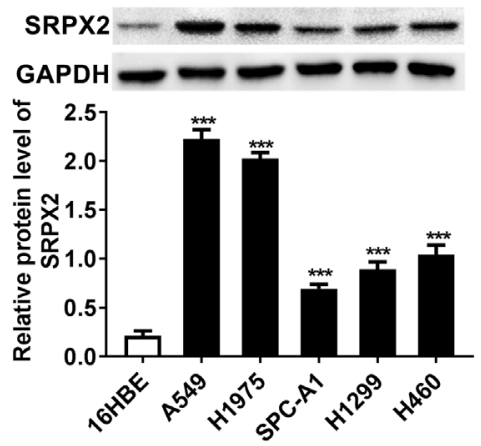

Figure 1. SRPX2 is upregulated in NSCLC tissues and associated with poor prognosis.

(A) qRT-PCR analysis of SRPX2 mRNA expression in 46 paired NSCLC and normal-adjacent tissue samples. (B) The protein level of SRPX2 in NSCLC tissues and normal-adjacent tissues was measured by western blot $(n=5)$. (C) IHC staining of SRPX2 in NSCLC samples. (D) Kaplan-Meier curves for the cumulative survival rate in NSCLC patients according to the relative mRNA expression of SRPX2 (dichotomized at the median). (E-F) The mRNA and protein expression levels of SRPX2 in A549, H1975, SPC-A1, H1229 and H460 cell lines and normal $16 \mathrm{HBE}$ cells were measured by GRT-PCR and western blot. GAPDH served as an endogenous control. All data were presented as mean \pm S.D.; data represent three independent experiments. ${ }^{* *} P<0.001$.

ethical guidelines for animal experiments. 5-week-old female BALB/c nude mice were obtained from Shanghai Experimental Animals Centre (Shanghai, China) and randomly divided into two groups: sh-NC and sh-SRPX2. A549 cells $\left(5 \times 10^{6}\right)$ transfected with sh-NC or sh-SRPX2 were injected subcutaneously into either flank of the mouse ( $n=5$ per group). The tumor width and length were measured every 3 days and the tumor volume was calculated using the formula: tumor volume $\left(\mathrm{mm}^{3}\right)=$ length $(\mathrm{mm}) \times$ width $(\mathrm{mm})^{2} \times 0.5$. After 21 days, mice were euthanized and tumors were excised and weighed. The tumor tissues were fixed for subsequent IHC assay.

Statistical analysis. Data were analyzed using a Statistical product (SPSS, version 20.0, SPSS Inc., Chicago, Illinois, USA) and presented as Mean \pm S.D. from three independent experiments. Overall survival curves were analyzed by Kaplan-Meier method and log-rank test. Comparison between two or more groups was performed by student's t-test or one-way ANOVA, respectively. $P<0.05$ was regarded to indicate a statistically significant difference.

\section{RESULTS}

SRPX2 is upregulated in NSCLC tissues and associated with poor prognosis

To confirm the dysregulation of SRPX2 in NSCLC, the mRNA level of SRPX2 was analyzed by qRT-PCR. Results (Fig. 1A) indicated that SRPX2 was considerably upregulated in NSCLC tissues (Tumor) compared to the adjacent non-tumor tissues (Non-tumor). Similarly, western blot and IHC assays also confirmed the upregulation of SRPX2 in NSCLC tissues compared to that in non-tumor tissues (Fig. 1B-C). Further statistical analysis on the relationship between SRPX2 expression and clinicopathological data in NSCLC patients was summarized in Table 1. The results revealed that the upregulation of SRPX2 was related to tumor size $(P=0.021)$, lymph node metastasis $(P=0.015)$, distant metastasis $(P=0.038)$ and clinical stage $(P=0.027)$ in NSCLC patients $(n=46)$. The correlation between SRPX2 expression and prognosis of NSCLC patients was analyzed via Kaplan-Meier survival 
Table 1. Correlation between expression of SRPX2 and clinicopathological Characteristics in NSCLC patients ( $n=46)$.

\begin{tabular}{|c|c|c|c|c|}
\hline \multirow[t]{2}{*}{ Variables } & \multirow{2}{*}{$\mathrm{n}$} & \multicolumn{2}{|l|}{ SRPX2 } & \multirow[t]{2}{*}{$P$ value } \\
\hline & & High expression $(n=25)$ & Low expression $(n=21)$ & \\
\hline \multicolumn{5}{|l|}{ Gender } \\
\hline Male & 16 & 9 & 7 & \\
\hline Female & 30 & 16 & 14 & 0.850 \\
\hline \multicolumn{5}{|l|}{ Age(years) } \\
\hline$\geq 65$ & 18 & 9 & 9 & \\
\hline$<65$ & 28 & 16 & 12 & 0.635 \\
\hline \multicolumn{5}{|l|}{ Smoking } \\
\hline Yes & 19 & 11 & 8 & \\
\hline No & 27 & 14 & 13 & 0.685 \\
\hline \multicolumn{5}{|l|}{ Pathology } \\
\hline Squamous cell carcinoma & 18 & 10 & 8 & \\
\hline adenocarcinoma & 26 & 14 & 12 & \\
\hline Others & 2 & 1 & 1 & 1.000 \\
\hline \multicolumn{5}{|l|}{ Differentiation } \\
\hline Well differentiated & 11 & 5 & 6 & \\
\hline Moderately differentiated & 18 & 9 & 9 & \\
\hline Lowly or undifferentiated & 17 & 11 & 6 & 0.543 \\
\hline \multicolumn{5}{|l|}{ T classification } \\
\hline $\mathrm{T} 1+\mathrm{T} 2$ & 29 & 12 & 17 & \\
\hline $\mathrm{T} 3+\mathrm{T} 4$ & 17 & 13 & 4 & 0.021 \\
\hline \multicolumn{5}{|l|}{ N classification } \\
\hline $\mathrm{N} 0+\mathrm{N} 1$ & 31 & 13 & 18 & \\
\hline $\mathrm{N} 2+\mathrm{N} 3$ & 15 & 12 & 3 & 0.015 \\
\hline \multicolumn{5}{|l|}{ Distant metastasis } \\
\hline M1 & 5 & 5 & 0 & \\
\hline Mo & 41 & 20 & 21 & 0.038 \\
\hline \multicolumn{5}{|l|}{ Clinical stage } \\
\hline$I+I I$ & 27 & 11 & 16 & \\
\hline III+IV & 19 & 14 & 5 & 0.027 \\
\hline
\end{tabular}

Bold values signifies $P<0.05$

analysis. As depicted in Fig. 1D, the high expression of SRPX2 correlated with significantly lower overall survival (OS) than the low expression of SRPX2 $(P=0.0086)$. These results revealed that high SRPX2 expression predicted poor prognosis of patients with NSCLC. SRPX2 was also upregulated in NSCLC cell lines (A549, H1975, SPC-A1, H1229 and H460) as compared to human bronchial epithelioid cell line 16HBE (Fig. 1E-F). A549 cells with higher expression of SRPX2 were selected for the subsequent loss-of-function assays, while SPC-A1 cells with lower expression of SRPX2 were used for gain-offunction assays.

\section{SRPX2 promotes proliferation in NSCLC cells}

The transfection efficiency of SRPX2 in SPC-A1 and A549 cells was validated by western blot assay. As illustrated in Fig. 2A, SRPX2 group displayed a significant increase in SRPX2 levels, indicating the successful transfection. Sh-SRPX2\#1 group with a higher SRPX2 knockdown efficiency was chosen for the subsequent loss-of-function assays and named as sh-SRPX2. CCK-8 and colony formation assays demonstrated that upregulation of SRPX2 dramatically promoted the proliferation of SPC-A1 cells, whereas knockdown of SRPX2 significantly decreased the proliferation of A549 cells (Fig. 2BC). Taken together, these data uncovered that SRPX2 might contribute to the progression of NSCLC by increasing cell proliferation.

\section{SRPX2 promotes migration and invasion of NSCLC cells}

As presented in Fig. 3A, wound healing assay revealed that the migratory capacity of SPC-A1 cells was evidently increased by SRPX2 overexpression, whereas the migration of A549 cells was reduced by SRPX2 knockdown. The transwell assay showed a significantly increased number of invasive cells when SRPX2 was overexpressed, whereas SRPX2 knockdown led to significantly decreased number of invasive cells (Fig. 3B). These data indicated that SRPX2 promoted NSCLC cell migration and invasion. 
A

SPC-A1

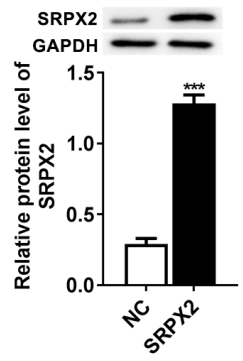

C

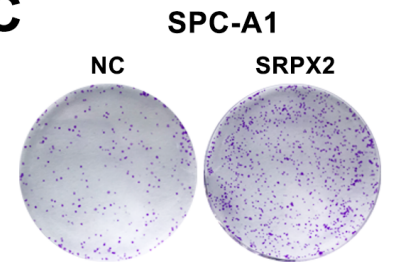

A549
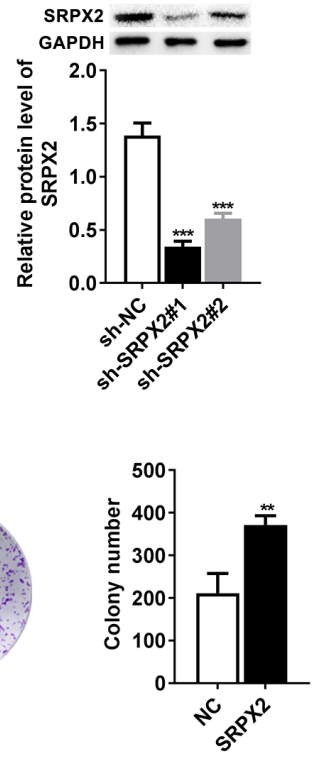

B
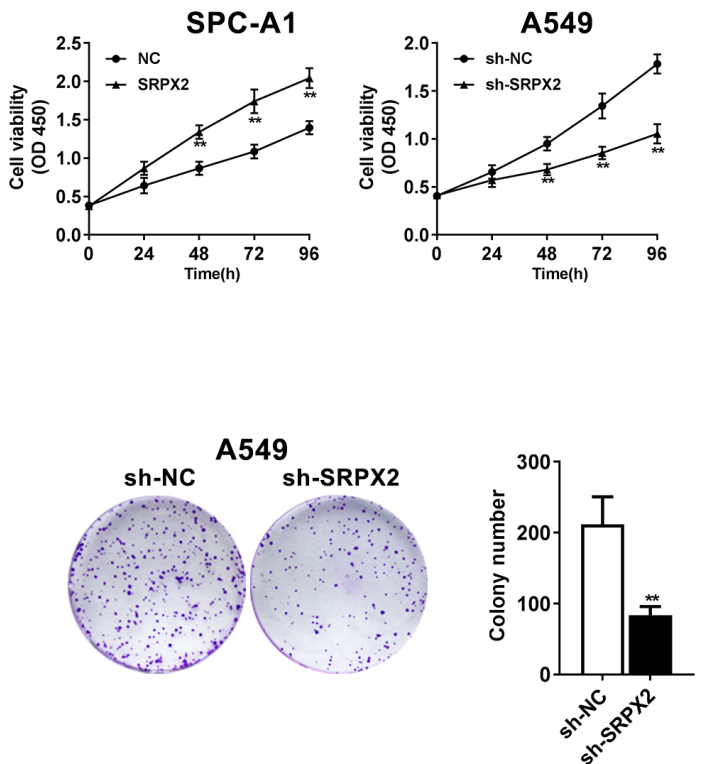

Figure 2. SRPX2 promotes proliferation of NSCLC cells.

(A) Transfection efficiency of SRPX2 overexpression vector in NSCLC cell lines, SPC-A1 and A549, was evaluated by western blot. (B) The proliferation of SPC-A1 and A549 cells was assessed by CCK-8 assay 24, 48, 72 and $96 \mathrm{~h}$ after transfection. (C) Colony formation assay was performed 2 weeks after transfection of SPC-A1 and A549 cells. All data were presented as mean \pm S.D.; data represent three independent experiments. ${ }^{* *} P<0.01 ;{ }^{* * *} P<0.001$.

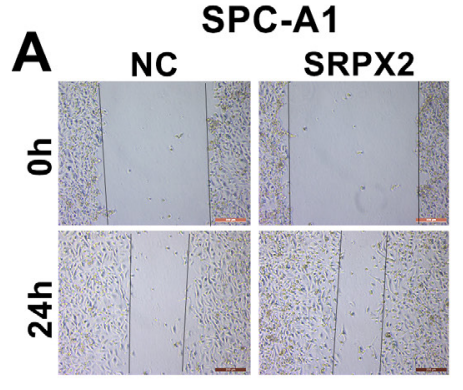

SPC-A1

B

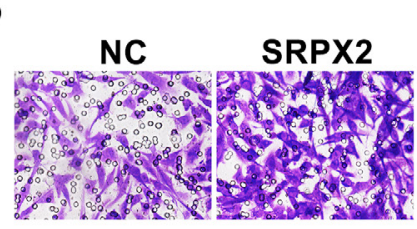

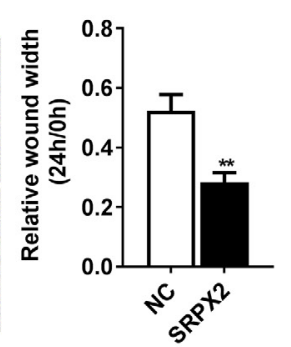

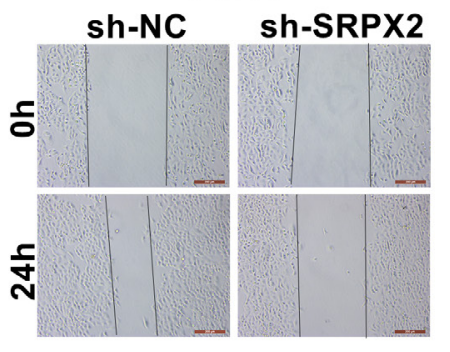

A549

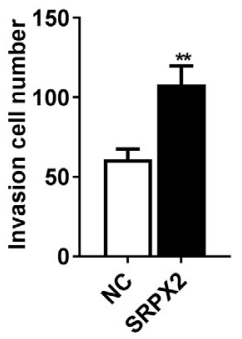

A549
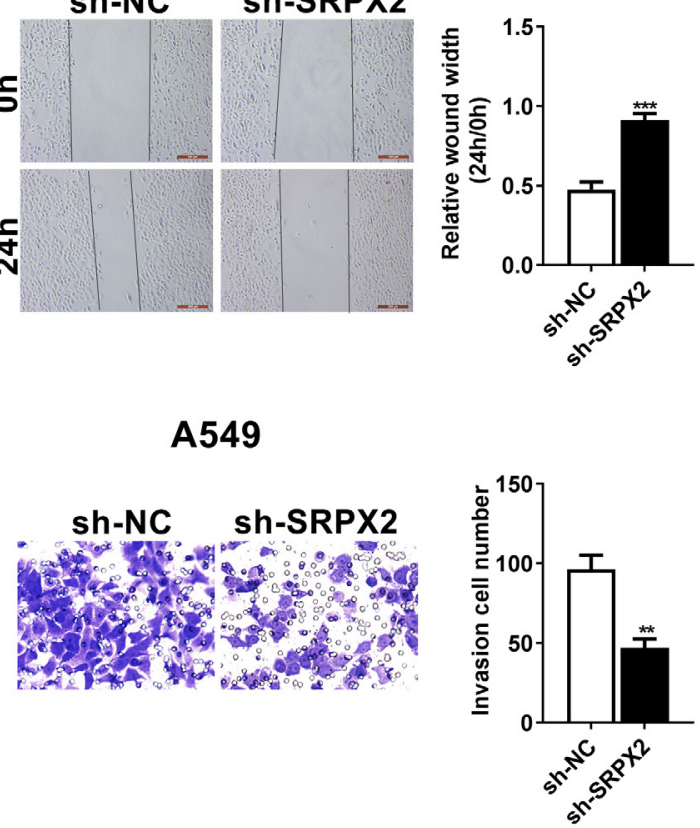

Figure 3. SRPX2 promotes migration and invasion of NSCLC cells.

(A) The migration of SPC-A1 and A549 cells was measured by wound healing assay. 0 and $24 \mathrm{~h}$ after scratch, the cells were photographed using a microscope. (B) The invasion of SPC-A1 and A549 cells was assessed by transwell assay $24 \mathrm{~h}$ after transfection. All data were presented as mean \pm S.D.; data represent three independent experiments. ${ }^{* *} P<0.01 ;{ }^{* *} P<0.001$.

SRPX2 modulates EMT and FAK/SRC/ERK pathway in NSCLC cells

Further analysis compared the levels of cell growthand EMT-related proteins in SPC-A1 and A549 cells. Overexpression of SRPX2 increased the protein levels of PCNA and N-cadherin and decreased the protein level of E-cadherin (Fig. 4A). Meanwhile, knockdown of SRPX2 caused the opposite results (Fig. 4B). As presented in Fig. 4A and 4B, overexpression of SRPX2 dramatically increased the phosphorylation levels of FAK (p-FAK), SRC (p-SRC) and ERK1/2 (p-ERK1/2), while the loss of SRPX2 decreased the phosphorylation levels 
A

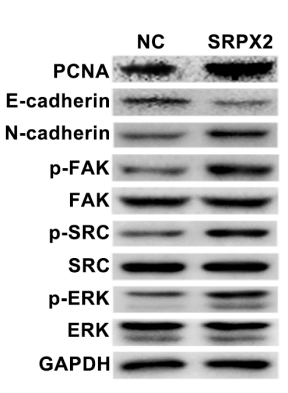

SPC-A1

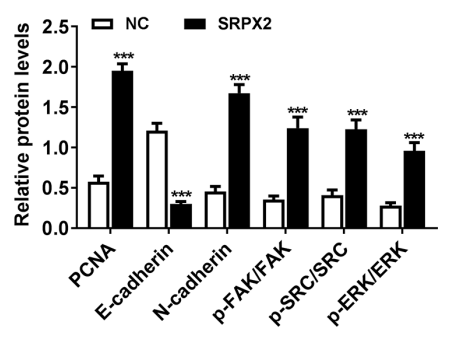

B

A549

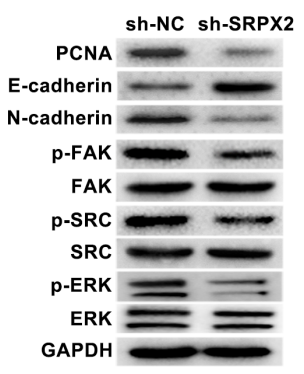

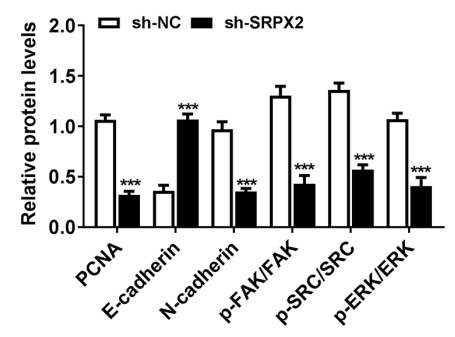

Figure 4. SRPX2 modulates EMT and FAK/SRC/ERK pathway.

(A-B) The protein levels of PCNA, E-cadherin, N-cadherin, p-FAK, FAK, p-SRC, SRC, p-ERK and ERK in SPC-A1 and A549 cells were measured by western blot $24 \mathrm{~h}$ after transfection. GAPDH served as an endogenous control. All data were presented as the mean \pm S.D.; data represent three independent experiments. ${ }^{* *} P<0.001$.
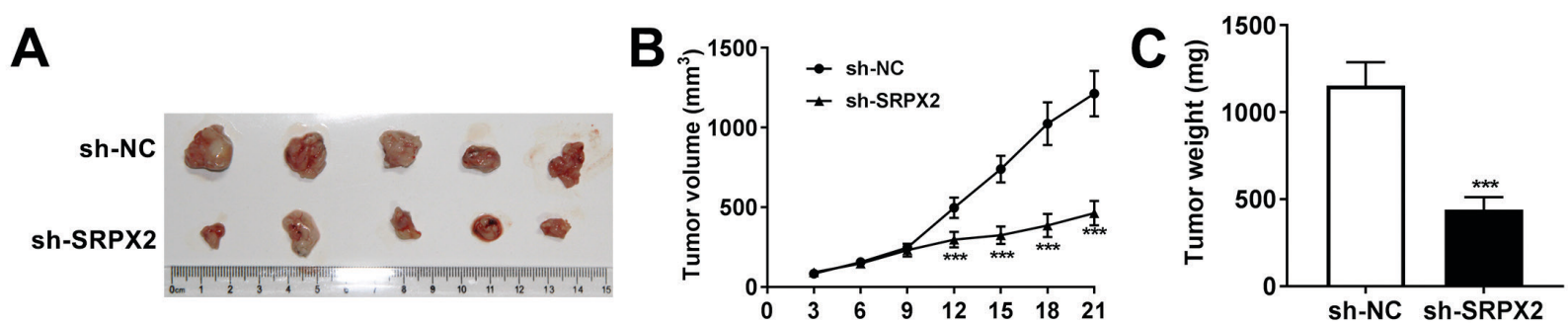

D

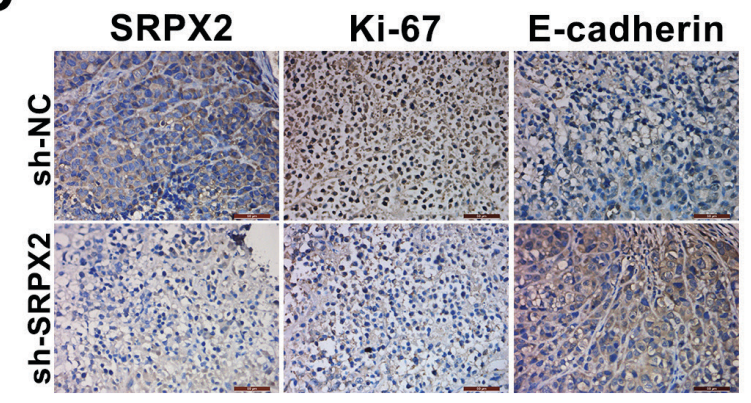

Figure 5. SRPX2 promotes tumor growth in xenograft mice.

(A) A549 cells transfected with sh-NC or sh-SRPX2 were hypodermically injected into either flank of the mouse ( $\mathrm{n}=5$ per group). After 21 days, the tumor nodules were photographed. (B-C) Tumor weight and volume were measured every 3 days after injection. (D) The expression of SRPX2, Ki-67 and E-cadherin in tumors was determined by IHC. All data were presented as the mean \pm S.D.; data represent three independent experiments. ${ }^{* * *} P<0.001$.

of these proteins. Therefore, these data indicated that SRPX2 promoted EMT and activated FAK/SRC/ERK signaling in NSCLC.

\section{SRPX2 promotes tumor growth in xenograft mice}

A549 cells with stable knockdown of SRPX2 were inoculated into nude mice. 21 days later, the subcutaneous tumors were harvested, and sh-SRPX2 group had smaller tumors than sh-NC group (Fig. 5A). The volume and weight of tumors were also obviously decreased in sh-SRPX2 group as compared with the sh-NC group (Fig. 5B-C). IHC staining (Figure 5D) indicated that the expression of SRPX2 and Ki67 were decreased while E-cadherin was increased in the sh-SRPX2 group. In conclusion, these results proved the functional role of SRPX2 in NSCLC tumorigenicity in vivo.

\section{DISCUSSION}

This is the first evidence confirming that SRPX2 is up-regulated in NSCLC tissues and cells, and high SRPX2 expression predicts poor prognosis and is associated with a range of clinicopathological characteristics, including tumor size, lymph node metastasis, distant metastasis and clinical stage. Moreover, we found that SRPX2 could promote NSCLC cell proliferation, migration and invasion in vitro, and promoted tumor growth in vivo. Interestingly, further experiments displayed that SRPX2 regulated the development of NSCLC via activating FAK/SRC/ERK pathway.

Lung cancer is surrounded by extensive ECM in both primary and metastatic sites, and is associated with poor prognosis (LIM et al., 2017). ECM provides spatial support for cell-to-cell and cell-to-matrix interac- 
tions (Lochter \& Bissell, 1995; Radisky \& Bissell, 2002). Numerous studies showed that ECM and its receptors are involved in the continuous progression of malignant transformation and metastasis (Ou et al., 2014; Lu, Weaver 7 Werb, 2012; Netti et al., 2000). The poor prognosis of NSCLC patients results from tumor metastasis and recurrence, which are closely related to the increased cancer cell mobility (Bremnes et al., 2002; He et al., 2018). Therefore, exploring the molecular mechanism is helpful for the therapy and diagnosis of NSCLC .

SRPX2, having the molecular mass of $53 \mathrm{kD}$, is located on chromosome Xq22.1 and is a downstream target gene of the E2A-HLF fusion gene (Sia et al., 2013; Kurosawa et al., 1999). Wilson identified SRPX2 as a chondroitin sulfate proteoglycan with the feature of ECM protein (Tanaka et al., 2012; Wilson et al., 2012). SRPX2 is abnormally expressed in a variety of tumors and could be used as a marker for tumor diagnosis. Lin et al. found that SRPX2 was highly expressed in and acted as an independent prognostic predictor of hepatocellular carcinoma (Lin et al., 2017). In addition, SRPX2 was also identified as a prognostic biomarker for prostate cancer (Zhang et al., 2018), pancreatic cancer (Li et al., 2019), gastric cancer (Tanaka et al., 2009), and glioblastoma (Tang et al., 2016).

More specifically, several previous studies indicated that SRPX2 exhibits carcinogenic function and was considered to be associated with malignant progression via different regulatory mechanisms. Gao and others (Gao et al., 2015). demonstrated that silencing of endogenous SRPX2 expression reduces migration and invasion of pancreatic carcinoma cells by regulating FAK-dependent signaling SRPX2 was also proved to promote the migration and invasion of hepatoma carcinoma cells by regulating the FAK/AKT signaling (Lin et al., 2017). Moreover, SRPX2 intensified the EMT process and promotes glioma metastasis through the MAPK signaling pathway (Tang et al., 2016). A recent study clarified the new mechanism of SRPX2 promoting the proliferation and metastasis and inhibiting the chemical sensitivity through inactivation of the $\mathrm{Wnt} / \beta$-catenin pathway in esophageal squamous cells (He et al., 2019). Nevertheless, no studies have considered the biological effect of SRPX2 on the development and progression of NSCLC. Our study indicated that SRPX2 could promote NSCLC cell proliferation, migration and invasion in vitro. In agreement with the in vitro results, knockdown of SRPX2 inhibited tumor growth and promoted the expression of epithelial marker (E-cadherin).

Focal adhesion kinase (FAK) and steroid receptor coactivator (c-Src) can regulate cytoskeleton dynamics and cell movement by affecting actin polymerization and focal adhesion inversion (Fife \& Kavallaris, 2014). Phosphorylated FAK and SRC form complexes that activate or inhibit multiple downstream signaling, including PI3K/AKT, P53, ERK etc. The FAK/SRC/ ERK signaling pathway plays important roles in the tumorigenesis and metastasis of various cancers, including NSCLC (Roy-Luzarraga \& Hodivala-Dilke, 2016; Patel et al., 2016; Kohno \& Pouyssegur, 2006). For instance, PIG3 enhances cell migration and invasion via promoting the FAK/Src pathway in lung adenocarcinoma $(\mathrm{Gu}$ et al., 2018). Maclurin inhibits migration and invasion of NSCLC cells through repressing the Src/FAK-ERK- $\beta$ catenin signaling ( $\mathrm{Ku}$ et al., 2015). Our study identified SRPX2 as a regulator of $\mathrm{FAK} / \mathrm{SRC} / \mathrm{ERK}$ signaling, evidenced by the increased levels of p-FAK, p-SRC pERK, which was consistent with previous studies (Gao et al., 2015; Lin et al., 2017). Besides, SRPX2 enhanced the protein levels of PCNA and N-cadherin, inhibited Ecadherin protein expression. These data confirmed that SRPX2 activated FAK/SRC/ERK signaling, regulated cell growth, modulated EMT-related proteins, and finally promoted NSCLC progression.

To sum up, this study uncovered the biological significance of SRPX2 in the development and progression of NSCLC. SRPX2 overexpression increased NSCLC cell proliferation, migration and invasion, which partly relied on the activation of FAK/SRC/ERK signaling. These results indicated that proteins of SRPX2-FAK/SRC/ ERK axis may be potential therapeutic targets and prognostic markers for NSCLC.

\section{Conflicts of interest}

There was no conflict of interest.

\section{Availability of data and materials}

All data generated or analyzed in this study are included in this article.

\section{Authors' contributions}

Xiujuan Li, Jing Liu and Hong Sun conceived and designed the experiments, Yong Zou, Juan Chen and Yichun Chen analyzed and interpreted the results of the experiments, Cheng Chen and Xuan Wu performed the experiments.

\section{Ethics approval and consent to participate}

The animal use protocol had been reviewed and approved by the Ethics Committees of Wuhan NO.1 Hospital.

\section{Patient consent for publication}

Not Applicable.

\section{REFERENCES}

Bremnes RM, Veve R, Hirsch FR, Franklin WA (2002) The E-cadherin cell-cell adhesion complex and lung cancer invasion metastasis and prognosis. Lung Cancer 36: 115-124. https://doi.org/10.1016/s0169$5002(01) 00471-8$

Caterson B (2012) Fell-Muir Lecture: Chondroitin sulphate glycosaminoglycans: fun for some and confusion for others. Int $J$ Exp Pathol 93: 1-10. https://doi.org/10.1111/j.1365-2613.2011.00807.x

Fife C, McCarroll J, Kavallaris M (2014) Movers and shakers: cell cytoskeleton in cancer metastasis. Br J Pharmacol 171: 5507-5523. https://doi.org/10.1111/bph.12704

Gao Z, Zhang J, Bi M, Han X, Han Z, Wang H, Ou Y (2015) SRPX2 promotes cell migration and invasion via FAK dependent pathway in pancreatic cancer. Int J Clin Exp Pathol 8: 4791

Gridelli C, Rossi A, Carbone DP, Guarize J, Karachaliou N, Mok T, Petrella F, Spaggiari L, Rosell R (2015) Non-small-cell lung cancer. Nat Rev Dis Primers 1: 15009. https://doi.org/10.1038/nrdp.2015.9

Gu MM, Gao D, Yao PA, Yu L, Yang XD, XingCG, Zhou J, Shang ZF, Li M (2018) p53-inducible gene 3 promotes cell migration and invasion by activating the FAK/Src pathway in lung adenocarcinoma. Cancer Sci 109: 3783. https://doi.org/10.1111/cas.13818

Gu X, Sun S, Gao X-S, Xiong W, Qin S, QiX, Ma M, Li X, Zhou D, Wang W (2016) Prognostic value of platelet to lymphocyte ratio in non-small cell lung cancer: evidence from 3430 patients. Sci Rep 6: 23893. https://doi.org/10.1038/srep23893

He F, Wang H, Li Y, Liu W, Gao X, Chen D, Wang Q, Shi G (2019) SRPX2 knockdown inhibits cell proliferation and metastasis and promotes chemosensitivity in esophageal squamous cell carcinoma. Biomed Pharmacotherapy 109: 671-678. https://doi.org/10.1016/j.biopha.2018.10.042

He W, Zhang H, Wang Y, Zhou Y, Luo Y, Cui N, Jiang W, Jiang H, Wang H, Xu D (2018) CTHRC1 induces non-small cell lung cancer (NSCLC) invasion through upregulating MMP-7/MMP-9. BMC Cancer 18: 400. https://doi.org/10.1186/s12885-018-4317-6 
Jemal A, RSiegel R, Xu J, Ward E (2010) Cancer statistics 2010. CA: Cancer Journal for Clinicians 60: 277-300. https://doi.org/10.3322/ caac. 21262

Jin H, Varner J (2004) Integrins: roles in cancer development and as treatment targets. Brit J Cancer 90: 561. https://doi.org/10.1038/ sj.bjc. 6601576

Kohno M, Pouyssegur J (2006) Targeting the ERK signaling pathway in cancer therapy. Ann Med 38: 200-211. https://doi. org/10.1080/07853890600551037

Ku MJ, Kim JH, Lee J, Cho JY, Chun T, Lee SY (2015) Maclurin suppresses migration and invasion of human non-small-cell lung cancer cells via anti-oxidative activity and inhibition of the Src/FAKERK- $\beta$-catenin pathway. Mol Cell Biochem 402: 243-252. https:// doi.org/10.1007/s11010-015-2331-4

Kurosawa $\mathrm{H}$, Goi K, Inukai T, Inaba T, Chang KS, Shinjyo T, Rakestraw KM, Naeve CW, Look AT (1999) Two candidate downstream target genes for E2A-HLF. Blood 93: 321-332

Li H, Zhang SR, Xu HX, Wang WQ, Li SS, Li TJ, Ni QX, Yu XJ, Liu L, Wu CT (2019) SRPX2 and RAB31 are effective prognostic biomarkers in pancreatic cancer. J Cancer 10: 2670-2678. https:// doi.org/10.7150/jca.32072

LIM SB, Tan SJ, Wan-Teck L, Lim CT (2017) An extracellular matrixrelated prognostic and predictive indicator for early-stage non-small cell lung cancer. Nat Commun 8: 1734. https://doi.org/10.1038/ s41467-017-01430-6

Lin X, Chang W, Wang Y, Tian M, Yu Z (2017) SRPX2 an independent prognostic marker promotes cell migration and invasion in hepatocellular carcinoma. Biomedicin Pharmacotherap 93: 398-405. https://doi.org/10.1016/j.biopha.2017.06.075

Liu K, Wu J, Zhou Y, Fan J (2015) Increased Sushi repeat-containing protein X-linked 2 is associated with progression of colorectal cancer. Med Oncol 32: 99. https://doi.org/10.1007/s12032-015-0548-4

Lochter A, Bissell MJ (1995). Involvement of extracellular matrix constituents in breast cancer. In Seminars in Cancer Biology. Lawrence Berkeley National Lab.(LBNL) Berkeley CA (United States)

Lomberk G (2010) The extracellular matrix and cell migration. Pancreatology 10: 4-5

Lu P, Weaver VM, Werb Z (2012) The extracellular matrix: a dynamic niche in cancer progression. I Cell Biol 196: 395-406. https://doi. org/10.1083/jcb.201102147

Malandrino A, Mak M, Kamm RD, Moeendarbary E (2018) Complex mechanics of the heterogeneous extracellular matrix in cancer. Extreme Mechanics Letters 21: 25-34. https://doi.org/10.1016/j. eml.2018.02.003

Naba A, Clauser KR, Lamar JM, Carr SA, Hynes RO (2014) Extracellular matrix signatures of human mammary carcinoma identify novel metastasis promoters. Elife 3: e01308. https://doi.org/10.7554/ eLife.01308

Netti PA, Berk DA, Swartz MA, Grodzinsky AJ, Jain RK (2000) Role of extracellular matrix assembly in interstitial transport in solid tumors. Cancer Res 60: 2497-2503

Øster B, Linnet L, Christensen LL, Thorsen K, Ongen H, Dermitzakis ET, Sandoval J, Moran S, Esteller M, Hansen TF (2013) Non-CpG island promoter hypomethylation and miR-149 regulate the expression of SRPX2 in colorectal cancer. Int J Cancer 132: 2303-2315. https://doi.org/10.1002/ijc.27921

Ou J, Peng Y, Deng J, Miao H, Zhou J, Zha L, Zhou R, Yu L, Shi H, Liang H (2014) Endothelial cell-derived fibronectin extra domain A promotes colorectal cancer metastasis via inducing epithelialmesenchymal transition. Carcinogenesis 35: 1661-1670. https://doi. org/10.1093/carcin/bgu090

Özbek S, Balasubramanian PG, Chiquet-Ehrismann R, Tucker RP, Adams JC (2010) The evolution of extracellular matrix. Mol Biol Cell 21: 4300-4305

Patel A, Sabbineni H, Clarke S, Somanath PR (2016) Novel roles of Src in cancer cell epithelial-to-mesenchymal transition vascular per- meability microinvasion and metastasis. Life Sci 157: 52-61. https:// doi.org/10.1016/j.lfs.2016.05.036

Pickup MW, Mouw JK, Weaver VM (2014) The extracellular matrix modulates the hallmarks of cancer. EMBO Reports 15: 1243-1253

Radisky D, Muschler J, Bissell MJ (2002) Order and disorder: the role of extracellular matrix in epithelial cancer. Cancer Invest 20: 139-153

Roll P, Rudolf G, Pereira S, Royer B, Scheffer IE, Massacrier A, Valenti M-P, Roeckel-Trevisiol N, Jamali S, Beclin C (2006) SRPX2 mutations in disorders of language cortex and cognition. Human Mol Genet 15: 1195-1207. https://doi.org/10.1093/hmg/ddl035

Rosso F, Giordano A, Barbarisi M, Barbarisi A (2004) From cell-ECM interactions to tissue engineering. J Cell Physiol 199: 174-180. https://doi.org/10.1002/jcp.10471

Roy-Luzarraga M, Hodivala-Dilke K (2016) Molecular pathways: endothelial cell FAK - a target for cancer treatment. Clin Cancer Res 22: 3718-3724. https://doi.org/10.1158/1078-0432.CCR-14-2021

Royer-Zemmour B, Ponsole-Lenfant M, Gara H, Roll P, Lévêque C, Massacrier A, FerracciG, Cillario J, Robaglia-Schlupp A, Vincentelli R (2008) Epileptic and developmental disorders of the speech cortex: ligand/receptor interaction of wild-type and mutant SRPX2 with the plasminogen activator receptor uPAR. Human Mol Genet 17: 3617-3630. https://doi.org/10.1093/hmg/ddn256

Rozario T, DeSimone DW (2010) The extracellular matrix in development and morphogenesis: a dynamic view. Develop Biol 341: 126140. https://doi.org/10.1016/j.ydbio.2009.10.026

Salmi M, Bruneau N, Cillario J, Lozovaya N, Massacrier A, Buhler E, Cloarec R, Tsintsadze T, Watrin F, Tsintsadze V (2013) Tubacin prevents neuronal migration defects and epileptic activity caused by rat Srpx2 silencing in utero. Brain 136: 2457-2473. https://doi. org/10.1093/brain/awt161

Sia G-M, Clem R, Huganir RL (2013) The human language-associated gene SRPX2 regulates synapse formation and vocalization in mice. Science 342: 987-991. https://doi.org/10.1126/science.1245079

Tanaka K, Arao T, Maegawa M, Matsumoto K, Kaneda H, Kudo K, Fujita Y, H. Yokote H, K. Yanagihara K, Y. Yamada Y (2009) SRPX2 is overexpressed in gastric cancer and promotes cellular migration and adhesion. Int J Cancer 124: 1072-1080. https://doi. org/10.1002/ijc. 24065

Tanaka K, Arao T, Tamura D, Aomatsu K, Furuta K, Matsumoto K, Kaneda H, Kudo K, Fujita Y, Kimura H (2012) SRPX2 is a novel chondroitin sulfate proteoglycan that is overexpressed in gastrointestinal cancer. PloS One 7: e27922. https://doi.org/10.1371/journal. pone. 0027922

Tang H, Zhao J, Zhang L, Zhao J, Zhuang Y, Liang P (2016) SRPX2 enhances the epithelial-mesenchymal transition and temozolomide resistance in glioblastoma cells. Cell Mol Neurobiol 36: 1067-1076. https://doi.org/10.1007/s10571-015-0300-9

Venning FA, Wullkopf L, Erler JT (2015) Targeting ECM disrupts cancer progression. Frontiers Oncol 5: 224. https://doi.org/10.3389/ fonc. 2015.00224

Wickström SA, Radovanac K, Fässler R (2011) Genetic analyses of integrin signaling. Cold Spring Harbor Perspectives Biol 3: a005116. https://doi.org/10.1101/cshperspect.a005116

Wilson R, Norris EL, Brachvogel B, Angelucci C, Zivkovic S, Gordon L, Bernardo CB, Stermann J, Sekiguchi K, Gorman JJ (2012) Changes in the chondrocyte and extracellular matrix proteome during post-natal mouse cartilage development. Mol Cell Proteomics 11: M111. 014159. https://doi.org/10.1074/mcp.M111.014159

Youlden DR, Cramb SM, Baade PD (2008) The International Epidemiology of Lung Cancer: geographical distribution and secular trends. J Thoracic Oncol 3: 819-831. https://doi.org/10.1097/ JTO.0b013e31818020eb

Zhang M, Li X, Fan Z, Zhao J, Liu S, Zhang M, Li H, Goscinski MA, Fan H, Suo Z (2018) High SRPX2 protein expression predicts unfavorable clinical outcome in patients with prostate cancer. OncoTargets Therapy 11: 3149. https://doi.org/10.2147/OT'T.S158820 